DOE/ER/40561-260-INT96-19-03

\title{
Glauber Multiple Scattering Theory for the Photoproduction of Vector Mesons off Nuclei and the Role of the Coherence Length
}

\author{
Jörg Hüfner \\ Institut für Theoretische Physik der Universität \\ Philosophenweg 19, D-69120 Heidelberg, Germany \\ Boris Kopeliovich' \\ Institute for Nuclear Theory, University of Washington \\ Seattle, WA98195, USA \\ and \\ Max-Planck-Institut für Kernphysik \\ Postfach 103980, D-69029 Heidelberg, Germany \\ Jan Nemchikn \\ Dipartimento di Fisica Teorica, Università di Torino \\ I-10125, Torino, Italy
}

\begin{abstract}
The integrated cross section for the incoherent photoproduction of vector mesons on nuclei $\gamma^{*} A \rightarrow V X, X \neq A$, is calculated within Glauber theory and as a function of the photon energy. The inverse of the longitudinal momentum transfer is called coherence length $l_{c}$ and depends on the virtuality and the energy of the photon. $\mathrm{Nu}$ clear transmission factors strongly depend on $l_{c} / R_{A}\left(R_{A}\right.$ is the nuclear radius $)$ and this effect may interfere with the search for color transparency effects.
\end{abstract}

\footnotetext{
${ }^{*}$ On leave of absence from Joint Institute for Nuclear Research, Laboratory of Nuclear Problems, Dubna, 141980 Moscow Region, Russia. E-mail: bzk@dxnhd1.mpi-hd.mpg.de

${ }^{\dagger}$ On leave of absence from Institute of Experimental Physics SAV, Solovjevova 47, CS-04353 Kosice, Slovakia
} 
With the help of the new powerful accelerators like CEBAF and HERA, which produce high energy (real or virtual) photons, the investigation of the hadronic processes inside nuclei (like color transparency, for example) will make substantial progress. Photons are particularly suitable for these studies since the production vertex for hadronic probes inside nuclei is well known and the analysis of the results is simpler and more reliable. In this note we treat the photoproduction of vector mesons inside nuclei and their rescattering via strong interactions within Glauber multiple scattering theory [1]. This is a simple problem treated by a conventional well accepted method. Yet, interference effects, which have not been treated before lead to a non-trivial energy- and $Q^{2}$-dependence of the production cross section and to unexpected phenomena. Any search for unconventional effects, like color transparency, should should refer to our calculations as a base line.

We study the incoherent photoproduction of a vector meson $V$ on a nucleus $A, \gamma^{*} A \rightarrow$ $V X$, where $\mathrm{X}$ is any state of the target nucleus except the ground state $|0\rangle$. The transition operator is given in eikonal form as

$$
\Gamma_{A}^{\gamma V}\left(\vec{b} ;\left\{\vec{s}_{j}, z_{j}\right\}\right)=\sum_{j=1}^{A} \Gamma_{N}^{\gamma V}\left(\vec{b}-\vec{s}_{j}\right) e^{i q_{L} z_{j}} \prod_{k(\neq j)}^{A}\left[1-\Gamma_{N}^{V V}\left(\vec{b}-\vec{s}_{k}\right) \Theta\left(z_{k}-z_{j}\right)\right]
$$

where $\left\{\vec{s}_{j}, z_{j}\right\}$ denote the coordinates of the target nucleon $N_{j} . \Gamma_{N}^{\gamma V}$ is the vector meson photoproduction amplitude on a nucleon, while $\Gamma_{N}^{V V}$ describes the elastic scattering amplitude for the vector meson on a nucleon. The $\Theta$-function allows vector meson rescattering only on those nucleons $k$, which lie "behind" the production point, i.e. $z_{k}>z_{j}$. The momentum $q_{L}$, which appears in the phase factor in eq. (1) is the difference between the longitudinal momenta of the photon and of the produced vector meson

$$
q_{L}=p^{\gamma}-p_{V}^{L}=\frac{Q^{2}+M_{V}^{2}+\left(p_{V}^{T}\right)^{2}}{2 \nu} .
$$

The quantity $q_{L}$, which depends on the energy $\nu$ and the virtuality $Q^{2}$ of the photon plays the central role in our consideration. Its inverse $l_{c}=1 / q_{L}$ is called the coherence length. Because of the phase factor in eq. (1), the photon production amplitude on two nucleons with positions $\left|z_{i}-z_{f}\right|<l_{c}$ add up coherently, otherwise there is destructive interference. 
The incoherent cross section for the production of a vector meson with transverse momentum $p_{V}^{T}$ in a reaction, where the nuclear state changes from $|0\rangle$ to $|f\rangle(f \neq 0)$ is given in Glauber theory by

$$
\frac{d \sigma_{i n c}^{\gamma V}(0 \rightarrow f)}{d^{2} p_{V}^{T}}=\left|\int \frac{d^{2} b}{2 \pi} \exp \left(-i \vec{p}_{V}^{T} \vec{b}\right)\left\langle f\left|\Gamma_{A}^{\gamma V}(\vec{b})\right| 0\right\rangle\right|^{2},
$$

from which one obtains the total incoherent cross section $\sigma_{i n c}^{\gamma V}$ by integrating over $\vec{p}_{V}^{T}$ and summing over all final states $|f\rangle$ with the exclusion of the ground state:

$$
\sigma_{i n c}^{\gamma V}=\int d^{2} b\left[\left\langle\left. 0|| \Gamma_{A}^{\gamma V}(b)\right|^{2} \mid 0\right\rangle-\left|\left\langle 0\left|\Gamma_{A}^{\gamma V}\right| 0\right\rangle\right|^{2}\right]
$$

We introduce the simplifying assumption

$$
\Gamma_{N}^{\gamma V}(b)=\lambda_{V} \Gamma_{N}^{V V}(b)
$$

where $\lambda_{V}$ is a constant, which equals to $e / f_{V}$ in the vector dominance model. Eq. (5) is a good approximation as long as the widths of the profile functions $\Gamma_{N}^{\gamma V}$ and $\Gamma_{N}^{V V}$ are small compared to the nuclear radius. We also use the relations for the elementary scattering processes of a vector meson on a nucleon

$$
\begin{aligned}
& \frac{1}{2} \sigma_{\text {tot }}^{V N}=\int d^{2} b \operatorname{Re} \Gamma_{N}^{V V}(b), \\
& \sigma_{e l}^{V N}=\int d^{2} b\left|\Gamma_{N}^{V V}(b)\right|^{2} .
\end{aligned}
$$

Furthermore, the nuclear wave function $|0\rangle$ is assumed to be a product of single particle wave functions and to be completely described by the density distribution $\rho(\vec{r})$, which is normalized to $A$. In order to bring out the essential effects, which are associated with the coherence length $l_{c}=1 / q_{L}$, we evaluate the incoherent cross section eq. (44) for two limiting cases, $q_{L} \rightarrow 0$ (high energy) and $q_{L} \rightarrow \infty$ (low energy), before we give the exact expression. For both limiting cases simple analytical formulae can be derived.

In the high energy limit, $q_{L} \rightarrow 0$, the phase factor $\exp \left(i q_{L} z\right)$ can be dropped in eq. (11), and after some algebra one finds 


$$
\Gamma_{A}^{\gamma V}\left(\vec{b} ;\left\{\vec{s}_{i}\right\}\right)=\lambda_{V}\left\{1-\prod_{j}\left[1-\Gamma_{N}^{V V}\left(\vec{b}-\vec{s}_{j}\right)\right]\right\}=\Gamma_{A}^{V V}\left(\vec{b} ;\left\{\vec{s}_{i}\right\}\right),
$$

where $\Gamma_{A}^{V V}$ is the Glauber amplitude for the scattering of the vector meson $V$ by the nucleus A. Using eqs. (41), (6) and (77), the incoherent cross section can be evaluated to give

$$
\sigma_{i n c}^{\gamma V}=\lambda_{V}^{2} \int d^{2} b\left[e^{-\sigma_{\text {in }}^{V N} T(b)}-e^{-\sigma_{\text {tot }}^{V N} T(b)}\right]
$$

where $T(b)=\int_{-\infty}^{\infty} d z \rho(b, z)$ is the nuclear thickness function, and $\sigma_{i n}^{V N}=\sigma_{\text {tot }}^{V N}-\sigma_{e l}^{V N}$. Note that eq. (8) has, up to the factor $\lambda_{V}^{2}$, the same form as the expression (see in [3]) for the cross section of quasielastic scattering of a hadron $(V)$ on a nucleus. In the low energy limit, when $q_{L} \gg R_{A}^{-1}\left(R_{A}\right.$ being the nuclear radius) all expressions which explicitly contain the phase factor go to zero, e.g.

$$
\left\langle 0\left|\Gamma_{A}^{\gamma V}\right| 0\right\rangle=0
$$

In the expression

$$
\begin{aligned}
& \left\langle\left. 0|| \Gamma_{A}^{\gamma V}\right|^{2} \mid 0\right\rangle=\lambda_{V}^{2}\langle 0| \sum_{i, j} \Gamma_{N}^{V V}\left(\vec{b}-\vec{s}_{i}\right) \Gamma_{N}^{V V^{*}}\left(\vec{b}-\vec{s}_{j}\right) e^{i q_{L}\left(z_{i}-z_{j}\right)} \times \\
& \prod_{k(\neq i)}\left[1-\Gamma_{N}^{V V}\left(\vec{b}-\vec{s}_{k}\right) \Theta\left(z_{k}-z_{i}\right)\right] \prod_{l(\neq j)}\left[1-\Gamma_{N}^{V V^{*}}\left(\vec{b}-\vec{s}_{l}\right) \Theta\left(z_{l}-z_{j}\right)\right]|0\rangle
\end{aligned}
$$

all terms with $i \neq j$ do not contribute to the matrix element and one is left with

$$
\sigma_{i n c}^{\gamma V}=\lambda_{V}^{2} \sigma_{e l}^{V N} \int d^{2} b \int_{-\infty}^{\infty} d z \rho(b, z) e^{-\sigma_{i n}^{V N} T_{z}(b)}
$$

where $T_{z}(b)=\int_{z}^{\infty} d z^{\prime} \rho\left(b, z^{\prime}\right)$.

The low and high energy limits, eqs. (11) and (8), respectively, were derived also in 4 . In order to expose the difference between these expressions we consider the case where the elastic $V N$ cross section is small $\sigma_{e l}^{V N} T(b) \ll 1$. Then we find 


$$
\sigma_{i n c}^{\gamma V}=\sigma\left(\gamma^{*} N \rightarrow V N\right) \int d^{2} b \int_{-\infty}^{\infty} d z \rho(b, z) \times \begin{cases}e^{-\sigma_{i n}^{V N} T_{z}(b)} & \text { (low energy }) \\ e^{-\sigma_{i n}^{V N} T(b)} & \text { (high energy) }\end{cases}
$$

where $\sigma\left(\gamma^{*} N \rightarrow V N\right)=\lambda_{V}^{2} \sigma_{e l}^{V N}$ is the photoproduction cross section on a nucleon. The two limiting cases, for low and high energies, respectively, differ in the attenuation factors. The integration in eq. (12) goes over all points $(b, z)$ for the photoproduction vertex $\gamma^{*} N \rightarrow V N$. In the low energy limit, the attenuation of the outgoing vector meson is governed by the $T_{z}(b)$, which is the nuclear thickness experienced by the vector meson from the point of creation $(\vec{b}, z)$ until its exit from the nucleus. This is what is expected.

At high energy, on the other hand, the attenuation is governed by $T(b)=T_{z=-\infty}(b)$, which is the thickness of the nucleus along the total path (the summed paths of the photon and the vector meson). The attenuation is governed by the same $\sigma_{i n}^{V N}$ independently of whether it acts on the incoming photon or the outgoing vector meson. We have to conclude that at high energies the photon has already converted to a virtual vector meson long before the vector meson is put on the energy shell via an interaction with a target nucleon at $(\vec{b}, z)$. This interpretation is familiar from the vector dominance model (VDM) [2], which predicts that at high energies a photon behaves in strong interactions like a hadron. Or the same phenomenon expressed in quantum mechanical language: because of the uncertainty principle a photon can convert virtually into a vector meson. The lifetime $\Delta t$ of such a fluctuation in the lab. frame is estimated to be $\Delta t \sim 1 / q_{L}$. If $\Delta t \gg 1 / R_{A}$ the chances are large that the incoming photon is already in the virtual state of a vector meson and therefore experiences a strong absorption before the fluctuation is put on the energy shell.

Note that we have not used the vector dominance model, but only eq. (5), which is rather general. However, the laws of quantum mechanics have been properly accounted for in eq. (11). Taking all the interference terms in the multiple scattering series into account leads to the different attenuations at low and high energies, i.e. for $q_{L} \rightarrow \infty$ and $q_{L} \rightarrow 0$, respectively. 
What is the proper scale, which separates high and low energies? There are a two dimensioned scales in the multiple scattering series, the nuclear radius $R_{A}$, and the mean free path of the vector meson in nuclear matter $l_{\text {free }} \approx 1 / \sigma_{i n}^{V N} \rho_{0}(\approx 3 \mathrm{fm}$ for $\rho-$ meson $)$. Condition $l_{c} \ll L_{A}=\min \left\{R_{A} ; l_{\text {free }}\right\}$ defines low and $l_{c} \gg L_{A}$ high energies, where $l_{c}=1 / q_{L}$ can be interpreted as the length, which a quantum mechanical fluctuation $\gamma \rightarrow V \rightarrow \gamma$ travels if it has the speed of light. We call $l_{c}$ the coherence length.

The general expression for the incoherent photoproduction cross section on a nucleus can be also calculated from the expression eq. (4). The result is a somewhat lengthy expression

$$
\begin{aligned}
\sigma_{i n c}^{\gamma V}= & \sigma(\gamma N \rightarrow V N) \int d^{2} b\left\{\int_{-\infty}^{\infty} d z \rho(b, z) e^{-\sigma_{i n}^{V N} T_{z}(b)}+\right. \\
& \frac{1}{2} \frac{\sigma_{t o t}^{V N}}{\sigma_{e l}^{V N}}\left(\sigma_{i n}^{V N}-\sigma_{e l}^{V N}\right) \int_{-\infty}^{\infty} d z_{1} \rho\left(b, z_{1}\right) \int_{z_{1}}^{\infty} d z_{2} \rho\left(b, z_{2}\right) \times \\
& \cos \left[q_{L}\left(z_{2}-z_{1}\right)\right] \exp \left[-\frac{1}{2}\left(\sigma_{\text {in }}^{V N}-\sigma_{e l}^{V N}\right) T_{z_{2}}(b)-\frac{1}{2} \sigma_{t o t}^{V N} T_{z_{1}}(b)\right]- \\
& \left.\frac{1}{4} \frac{\left(\sigma_{\text {tot }}^{V N}\right)^{2}}{\sigma_{e l}^{V N}}\left|\int_{-\infty}^{\infty} d z \rho(b, z) e^{i q_{L} z} e^{-\frac{1}{2} \sigma_{t o t}^{V N}} T_{z}(b)\right|^{2}\right\},
\end{aligned}
$$

where the last term in the curly brakets is the cross section for coherent production $\gamma A \rightarrow$ $V A$ [2]. Formula (13) for incoherent photoproduction is new and is the main result of the present paper.

In the two limits $q_{L} \rightarrow 0$ and $q_{L} \rightarrow \infty$ formula([13) leads to expressions (8) and (11), respectively, as it should be. As an illustration we show numerical examples for the effect of the coherence length. We calculate the nuclear transmission function $\operatorname{Tr}^{\gamma V}\left(q_{L}, A\right)$, which is defined by

$$
\operatorname{Tr}^{\gamma V}\left(q_{L}, A\right)=\frac{\sigma_{i n c}\left(\gamma A \rightarrow V X ; q_{L}\right)}{A \sigma(\gamma N \rightarrow V N)}
$$

for the incoherent photoproduction of $\rho$-mesons as a function of the energy. The results for carbon, iron and lead are shown in Fig. 1 (one can find more examples in [5]) as a function of the energy $\nu$ and the virtuality $Q^{2}$ of the photon. Both quantities appear in $q_{L}$ eq. (2)). 
The transverse momentum $p_{V}^{T}$ in eq. (2) has been set equal zero. The cross sections which enter the calculation are $\sigma_{t o t}^{\rho N}=25 \mathrm{mb}$ and $\sigma_{e l}^{V N}=\left(\sigma_{t o t}^{V N}\right)^{2} / 16 \pi B_{e l}^{V N}$, where $B_{e l}^{\rho N} \approx 8 \mathrm{GeV}^{-2}$ is the slope of the differential cross section of elastic $\rho N$ scattering.

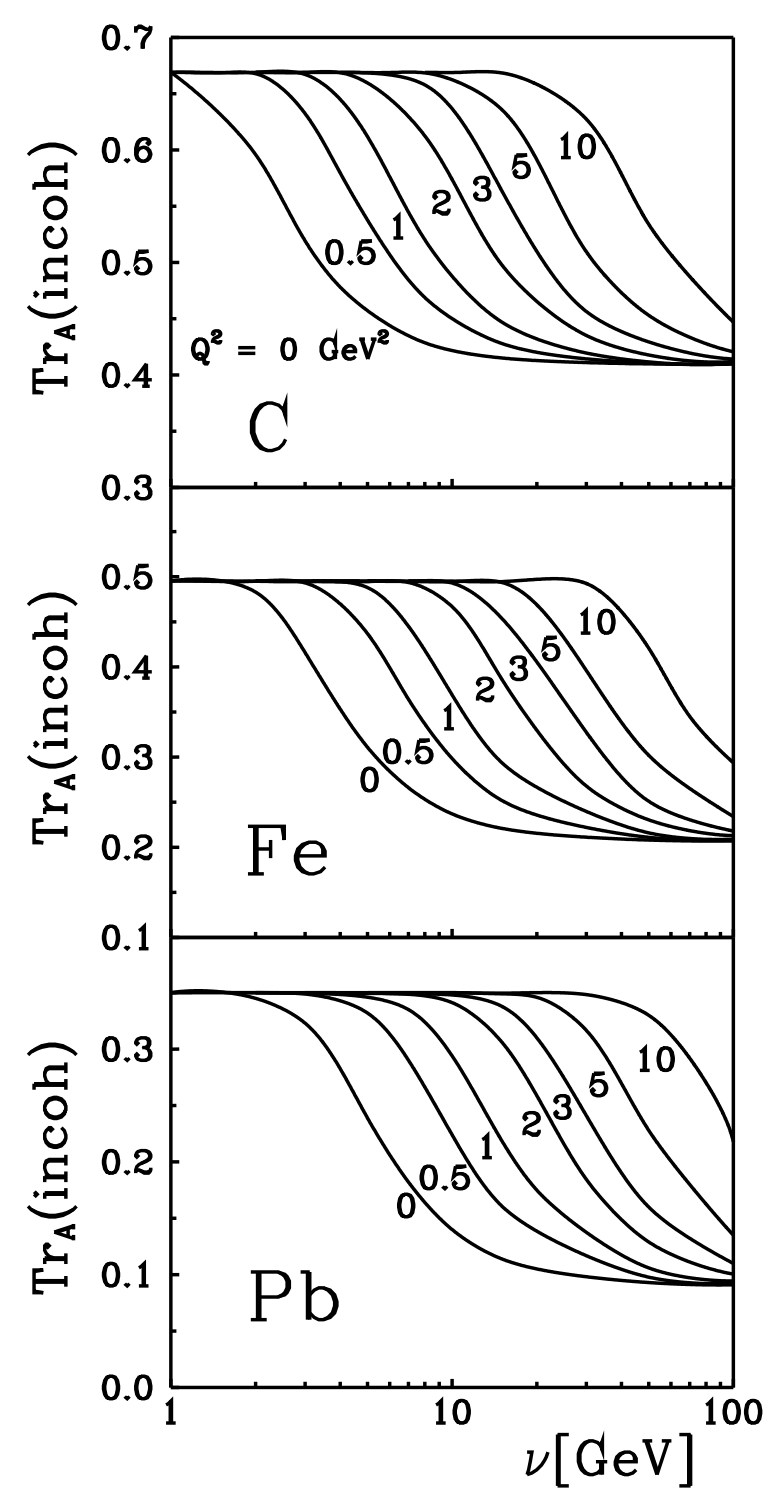

Figure 1: Nuclear transparency for incoherent photoproduction of $\rho$ mesons on $C, F e$ and $P b$ nuclei as a function of the photon energy $\nu$ and for values $Q^{2}$ of the virtuality of the photon between 0 and $10(\mathrm{GeV} / \mathrm{c})^{2}$.

The numerical results in Fig. 1 display a strong variation of the nuclear transparency as a function of $\nu$ and $Q^{2}$. The numerical values in the low and high energy limits $\left(q_{L} \rightarrow \infty\right.$ 
and $q_{L} \rightarrow 0$, respectively obey the approximate relation

$$
\operatorname{Tr}\left(q_{L} \rightarrow 0\right) \approx\left[\operatorname{Tr}\left(q_{L} \rightarrow \infty\right)\right]^{2}
$$

The half value between the low and high energy limits is reached for $\left(q_{L} \cdot R_{A}\right) \simeq 1.5$, where $R_{A}=1.2 A^{1 / 3} \mathrm{fm}$.

Although eq. (13) can be evaluated numerically in a rather straightforward way, as is done for Fig. 1, we have also looked for approximate expressions for the nuclear transparency. In the limit of small attenuation, $\sigma_{i n}^{V N} \cdot\langle T\rangle \ll 1$, the transparency becomes

$$
\operatorname{Tr}^{\gamma V}\left(q_{L}, A\right) \approx\left\{1-\frac{1}{2} \sigma_{i n}^{V N}\langle T\rangle\left[1+F_{A}^{2}\left(q_{L}\right)\right]\right\}
$$

where $F_{A}$ is the nuclear formfactor

$$
F_{A}^{2}\left(q_{L}\right)=\frac{1}{A\langle T\rangle} \int d^{2} b\left|\int_{-\infty}^{\infty} d z \rho(b, z) e^{i q_{L} z}\right|^{2},
$$

and

$$
\langle T\rangle=\frac{1}{A} \int d^{3} r \rho(\vec{r}) T(b)
$$

is the mean nuclear thickness. For $q_{L} R_{A} \gg 1$ (low energy) one has $F_{A} \rightarrow 0$, but $F_{A} \rightarrow 1$ if $q_{L} R_{A} \ll 1$ (high energy). The corresponding transparencies differ by a factor of two in the second term in the square brackets in eq. (16). The approximate formulae may be good for $J / \psi$ photoproduction as it was suggested in [6], but fails for the $\rho$-meson.

We have presented a fully quantum mechanical derivation for the incoherent photonproduction of vector mesons off nuclei using Glauber's formalism of multiple scattering theory. Strong energy and $Q^{2}$ dependencies are predicted, which arise from interference effects, which essentially depend on the coherence length $l_{c}=1 / q_{L}$. For low energies, $l_{c} \rightarrow 0$, one recovers the classical picture of a vector meson produced inside the nucleus and attenuated on its way out. In the other limit of high energies, $l_{c} \rightarrow \infty$, the incoming photon is already a virtual vector meson and thus experiences attenuation before it converts to the on-shell meson. This strong dependence on $q_{L}$ leads to a rising $Q^{2}$-dependence at fixed energy (see examples in [5]) and has to be accounted for before one may look for effects like color transparency. 
Acknowledgement: The work was partially supported by a grant from the Federal Ministry for Education and Research (BMBF), grant number 06 HD 742, and by INTAS grant 93-0239. B.K. thanks the Institute for Nuclear Theory at the University of Washington for its hospitality and the Department of Energy for partial support during the completion of this work.

\section{References}

[1] R.J. Glauber, In: Lectures in Theor. Phys., v. 1, ed. W.E. Brittin and L.G. Duham. NY: Intersciences, 1959

[2] T.H. Bauer, R.D. Spital, D.R. Yennie and F.M. Pipkin, Rev. Mod. Phys. 50 (1978) 261

[3] A.V. Tarasov, Fiz.Chastits I Yadra (Sov. Phys) 7 (1976) 771

[4] B.Z. Kopeliovich and B.G. Zakharov, Phys.Lett. B264 (1991) 434

[5] B.Z. Kopeliovich and J. Nemchik, Where is the Baseline for Color Transparency Studies with Moderate Energy Electron Beams?, MPIH-V41-1995, nucl-th/9511018

[6] O. Benhar, B.Z. Kopeliovich, Ch. Mariotti, N.N. Nikolaev and B.G. Zakharov, Phys. Rev. Lett. 69 (1992) 1156 